\title{
Adsorption of chain molecules in pores with crystalline walls: a density functional approach
}

\author{
K.Bucior ${ }^{1}$, A.Patrykiejew ${ }^{1}$, S.Sokołowski ${ }^{1}$, O.Pizio ${ }^{2}$ \\ 1 Department for the Modelling of Physico-Chemical Processes, Maria Curie-Skłodowska University, \\ 20-031 Lublin, Poland \\ 2 Universidad Autonoma Metropolitana, Iztapalapa, México D.F., México
}

Received November 29, 2008, in final form February 7, 2009

\begin{abstract}
A microscopic density functional theory of adsorption of chain molecules on the attractive solid surfaces of crystalline symmetry is developed. The proposed approach is based on the expansions of one-particle functions into two-dimensional Fourier series. Illustrative calculations for dimers, 8- and 16-mers adsorbed on a (100) fcc crystal surface indicate the effect of the periodicity of the adsorbing potential on the structure of the adsorbed layer and thermodynamics of adsorption.
\end{abstract}

Key words: density functional, adsorption, chains, crystals

PACS: 68.08.-p, 68.43.Fg, 82.35.Gh, 68.43.-h

\section{Introduction}

Physical adsorption of nonspherical molecules on well defined crystal surfaces has been a field of vivid activity for several years [1-11]. These studies indicated that periodic modulation of an adsorbing potential may cause strong orientational effects in adsorbed films formed by linear molecules on the surfaces of square and rectangular lattice symmetry [12-16]. Compared to the films composed of spherical adsorbates that already exhibit very complex phase behavior of adsorbed layers [17], the films formed by nonspherical molecules show many new and interesting features $[18,19]$. In a majority of hitherto published studies, the adsorbate particles were composed of a small number of atoms (or segments), most frequently two or three. Moreover, theoretical approaches were usually based on the application of computer simulation techniques, whereas analytical methods were rather seldom used [17-19].

Recently, several versions of a density functional approach for nonuniform molecular systems were proposed. Among them the theories, which extend Wertheim's thermodynamic perturbation theory [20] to the case of nonuniform chain fluids $[21,22]$, seem to be particularly convenient for the purpose of studying the structure and various thermodynamic properties of nonuniform systems composed of tangentially bonded chains [23-26].

The majority of previous studies of adsorption of chain particles using the density functional approaches were restricted to the models with structureless adsorbing surfaces. The aim of this work is to extend the density functional theory of $\mathrm{Yu}$ and $\mathrm{Wu}[21,22,25,26]$ to the case of crystalline surfaces. Our extension is performed by using the ideas from the earlier studies of adsorption of simple [27] and associating [28,29] fluids on crystalline surfaces. The present work concentrates on the methodological aspects of the theory. Our principal objective is to develop equations for the density profiles appropriate to the description of the fluid of chain molecules in contact with the surfaces that exhibit finite corrugation of the external field. Theoretical deliberations are enhanced by numerical results for simple model systems. Thermodynamic properties including the phase behavior of more complex inhomogeneous fluids in contact with crystalline walls will become the subject of future works. 


\section{Theory}

We consider a fluid of chain molecules composed of the same segments in contact with a single wall of a regular crystal, characterized by the unit lattice vectors $\mathbf{a}_{1}$ and $\mathbf{a}_{2}$. Each fluid molecule is built of $M$ hard spheres of diameter $\sigma$ each, which are tangentially jointed by a bonding potential acting between the adjacent segments. Similarly to the approach of Wu et. al. $[21,22,25,26]$ we assume that the bonding potential between the two nearest-neighbor segments, $v_{b}(r)$, is of infinitely short range, i.e.,

$$
\exp \left[-\beta v_{b}(r)\right]=\left(1 /\left(4 \pi \sigma^{2}\right)\right) \delta(r-\sigma) .
$$

The potential (1) forbids any configuration that would lead to an overlap between two segments. The total bonding potential, $V_{b}\left(\mathbf{r}_{1}, \mathbf{r}_{2}, \ldots, \mathbf{r}_{M}\right)$ is a sum of the bonding potentials $v_{b}$ between the segments, $V_{b}\left(\mathbf{r}_{1}, \mathbf{r}_{2}, \ldots, \mathbf{r}_{M}\right)=\sum_{j=1}^{M-1} v_{b}\left(\left|\mathbf{r}_{j+1}-\mathbf{r}_{j}\right|\right)$.

Each segment of the chain particle interacts with a single pore wall via the potential $v^{\prime}(\mathbf{r})$. This potential results from the summation of Lennard-Jones $(12,6)$ potentials

$$
u_{g s}(r)=4 \varepsilon_{g s}\left[\left(\sigma_{g s} / r\right)^{12}-\left(\sigma_{g s} / r\right)^{6}\right]
$$

over all solid atoms. In the above $r$ is the distance between a segment and an atom of the pore wall and $\varepsilon_{g s}$ and $\sigma_{g s}$ are the energy and the size parameters. Following Steele [30] we apply a two-dimensional Fourier representation of the potential $v^{\prime}(\mathbf{r})$,

$$
v^{\prime}(\mathbf{r})=\sum_{\mathbf{q}_{j} \geqslant \mathbf{0}} v_{\mathbf{q}_{j}}^{\prime}(z) \exp \left[-\mathrm{i} \mathbf{q}_{j} \cdot \mathbf{t}\right]
$$

where $z$ is the coordinate perpendicular to the surface, $\mathbf{q}_{j}=2 \pi \mathbf{b}_{1} j_{1}+\mathbf{b}_{2} j_{2}$ are the reciprocal lattice vectors, $j_{1}, j_{2}=0, \pm 1, \pm 2, \ldots$ and $\mathbf{t}=(x, y)$ is a two-dimensional vector parallel to the surface. The analytical expressions for the Fourier coefficients $v_{\mathbf{q}_{j}}(z)$ for crystal lattices of different symmetry can be found in [30]. Briefly, the coefficients $v_{\mathbf{q}_{j}}^{\prime}(z)$ depend only on the length of the vectors $\mathbf{q}_{j}$ and, therefore, one can define the functions $f_{j}(x, y)=\sum^{\prime} \exp \left[-\mathbf{i} \mathbf{q}_{j} \cdot \mathbf{t}\right]$, where the prime means that the summation runs over all reciprocal vectors of a given length $\left|\mathbf{q}_{j}\right|$. The functions $f_{j}(x, y)$ depend on the parameter $\sigma_{g s}$ of the potential (2), whereas the coefficients $v_{\left|\mathbf{q}_{j}\right|}(z)$ depend on $\sigma_{g s}$ and $\varepsilon_{g s}[30]$.

In the case of a slit-like pore of the width $H$, the potential of the segment-pore walls is the sum of the potentials (3) exerted by two pore walls. In a general case the crystalline symmetry of the walls may not be the same. Moreover, even for the same symmetry, there may be a shift between crystal lattices forming two walls. In this work we consider the simplest case of the pore walls of the same symmetry and assume that there is no phase shift between the segment-wall potentials, i.e.

$$
v(\mathbf{r})=\sum_{\mathbf{q}_{j} \geqslant \mathbf{0}}\left[v_{\mathbf{q}_{j}}^{\prime}(z)+v_{\mathbf{q}_{j}}^{\prime}(H-z)\right] \exp \left[-\mathbf{i} \mathbf{q}_{j} \cdot \mathbf{t}\right] .
$$

The density functional theory of adsorption of chain molecules developed in $[21,22,25,26]$ leads to the following equation for the density profile of the segment $j, j=1, \ldots, M$

$$
\rho_{s j}\left(\mathbf{r}_{j}\right)=\exp (\beta \mu) \int \mathrm{d} \mathbf{r}_{1} \mathrm{~d} \mathbf{r}_{2} \ldots \mathrm{d} \mathbf{r}_{M} \delta\left(\mathbf{r}-\mathbf{r}_{j}\right) \exp \left[-\beta V_{b}\left(\mathbf{r}_{1}, \mathbf{r}_{2}, \ldots, \mathbf{r}_{M}\right)\right] \prod_{l=1}^{M}\left[g\left(\mathbf{r}_{l}\right) \gamma\left(\mathbf{r}_{l}\right)\right]
$$

which, for identical segments and for the bonding potential given by equation (1), can also be rewritten as

$$
\tau_{s j}\left(\mathbf{r}_{j}\right)=\exp (\beta \mu) \gamma_{j}\left(\mathbf{r}_{j}\right) G^{j}\left(\mathbf{r}_{j}\right) G^{M+1-j}\left(\mathbf{r}_{j}\right)
$$

where $\tau_{s j}(\mathbf{r})$ is one-particle cavity function, $\rho_{s j}(\mathbf{r})=\tau_{s j}(\mathbf{r}) g(\mathbf{r}), g(\mathbf{r})=\exp [-\beta v(\mathbf{r})]$ and the functions $G^{l}(\mathbf{r})$ are given by the recurrence relation

$$
G^{l}(\mathbf{r})=(1 /(4 \pi \sigma)) \int \mathrm{d} \mathbf{r}^{\prime} \delta\left(\left|\mathbf{r}-\mathbf{r}^{\prime}\right|-\sigma\right) G^{l-1}\left(\mathbf{r}^{\prime}\right) \gamma_{l}\left(\mathbf{r}^{\prime}\right) \quad \text { for } \quad l \geqslant 2 .
$$


and $G^{1}(\mathbf{r}) \equiv 1$. In the above $\mu$ is the chemical potential of the fluid and $\gamma_{i}(\mathbf{r})=\exp \left[-\beta \lambda_{i}(\mathbf{r})\right]$,

$$
\lambda_{i}(\mathbf{r})=\frac{\delta F_{\mathrm{ex}}}{\delta \rho_{s i}(\mathbf{r})},
$$

with the excess free energy $F_{\mathrm{ex}}=\int \mathrm{d} \mathbf{r} \Phi_{\mathrm{ex}}(\mathbf{r}), \Phi_{\mathrm{ex}}(\mathbf{r})=\Phi_{\mathrm{HS}}(\mathbf{r})+\Phi_{\mathrm{C}}(\mathbf{r})$ being the functional of the free energy density due to hard-sphere interactions and chain connectivity and where $\rho_{s}(\mathbf{r})=$ $\sum_{j=1}^{M} \rho_{s j}(\mathbf{r})$ is the total segment density.

The free energy densities $\Phi_{\mathrm{HS}}$ and $\Phi_{\mathrm{C}}$ are given by

$$
\Phi_{\mathrm{HS}}=-n_{0} \ln \left(1-n_{3}\right)+\frac{n_{1} n_{2}-\mathbf{n}_{1} \cdot \mathbf{n}_{2}}{1-n_{3}}+n_{2}^{3}\left(1-\xi^{2}\right)^{3} \frac{n_{3}+\left(1-n_{3}\right)^{2} \ln \left(1-n_{3}\right)}{36 \pi n_{3}^{2}\left(1-n_{3}\right)^{2}},
$$

and

$$
\Phi_{\mathrm{C}}=\frac{1-M}{M} n_{0} \zeta \ln \left[\frac{1}{1-n_{3}}+\frac{n_{2} \sigma \zeta}{4\left(1-n_{3}\right)^{2}}+\frac{\left(n_{2} \sigma\right)^{2} \zeta}{72\left(1-n_{3}\right)^{3}}\right],
$$

where $\xi(\mathbf{r})=\left|\mathbf{n}_{2}(\mathbf{r})\right| / n_{2}(\mathbf{r}), \zeta=1-\mathbf{n}_{2} \cdot \mathbf{n}_{2} /\left(n_{2}\right)^{2}$, and where $n_{\alpha}, \alpha=0,1,2,3$ and $\mathbf{n}_{\alpha}, \alpha=1,2$ are scalar and vector averaged densities,

$$
\begin{aligned}
& n_{\alpha}(\mathbf{r})=\int \mathrm{d} \mathbf{r}^{\prime} \rho_{s}\left(\mathbf{r}^{\prime}\right) w_{\alpha}\left(\mathbf{r}-\mathbf{r}^{\prime}\right) \quad \text { for } \quad \alpha=0,1,2,3, \\
& \mathbf{n}_{\alpha}(\mathbf{r})=\int \mathrm{d} \mathbf{r}^{\prime} \rho_{s}\left(\mathbf{r}^{\prime}\right) \mathbf{w}_{\alpha}\left(\mathbf{r}-\mathbf{r}^{\prime}\right) \quad \text { for } \quad \alpha=1,2,
\end{aligned}
$$

with the weight functions $w_{\alpha}$ and $\mathbf{w}$ given in [31]. Note that in equations (9) and (10) the spatial dependence of all variables has been omitted for the sake of brevity. For detailed derivation of the above equations and equation (6) see $[21,22,25,26]$.

In order to rewrite equation (6) in a form suitable for the case of a crystalline surface we realize that any one-particle function, $\chi(\mathbf{r})$ can be expanded into a two-dimensional Fourier series

$$
\chi(\mathbf{r})=\sum_{\mathbf{q}_{j}} \chi_{\mathbf{q}_{j}}(z) \exp \left[-\mathbf{i} \mathbf{q}_{j} \cdot \mathbf{t}\right],
$$

where

$$
\chi_{\mathbf{q}_{j}}(z)=\frac{1}{\mathbf{a}_{1} \times \mathbf{a}_{2}} \iint_{\mathbf{a}_{1} \times \mathbf{a}_{2}} \chi(\mathbf{r}) \exp \left[i \mathbf{q}_{j} \cdot \mathbf{t}\right] \mathrm{d} \mathbf{t} .
$$

The Fourier expansion coefficients $\chi_{\mathbf{q}_{j}}(z)$ depend only on the length of the reciprocal lattice vector, $\left|\mathbf{q}_{j}\right|$.

Using equations (6), (12) and (13), the expansion coefficients of the function $\tau_{s j}(\mathbf{r})$ can be related to the expansion coefficients $\gamma_{i, \mathbf{q}}(z)$ and $G_{\mathbf{q}}^{l}(z)$ via

$$
\tau_{s j, \mathbf{q}}(z)=\exp (\beta \mu) \sum_{\mathbf{q}_{a}+\mathbf{q}_{b}+\mathbf{q}_{c}=\mathbf{q}} \gamma_{i \cdot \mathbf{q}_{a}}(z) G_{\mathbf{q}_{b}}^{j}(z) G_{\mathbf{q}_{c}}^{M+1-j}(z),
$$

and the recurrence relation (7) becomes

$$
G_{\mathbf{q}}^{l}(z)=\frac{1}{2 \sigma} \sum_{\mathbf{q}_{a}+\mathbf{q}_{b}+\mathbf{q}_{c}=\mathbf{q}} \int_{z-\sigma}^{z+\sigma} \mathrm{d} z^{\prime} J_{0}\left(|\mathbf{q}| \sqrt{\sigma^{2}-\left(z-z^{\prime}\right)^{2}}\right) \gamma_{i, \mathbf{q}_{a}}\left(z^{\prime}\right) g_{\mathbf{q}_{b}}\left(z^{\prime}\right) G_{\mathbf{q}_{c}}^{l-1}\left(z^{\prime}\right),
$$

where $J_{0}(u)$ is the zeroth-order Bessel function of the first kind. Note that for the model in question the expansion coefficients $\gamma_{i, \mathbf{q}}\left(z^{\prime}\right)$ are identical for all segments $1 \leqslant i \leqslant M$.

The last two equations comprise the solution for the segment density profile of a chain fluid in contact with a crystalline surface. Their application, however, requires evaluating the Fourier coefficients of the Boltzmann function $g(\mathbf{r})$ and the function $\gamma(\mathbf{r})$, according to equation (13). In the case of the Boltzmann function the integration is instantaneous, whereas in order to calculate 
the coefficients $\gamma_{s j, \mathbf{q}}(z)$ one must first calculate the coefficients $\lambda_{s j, \mathbf{q}}(z)$. To do that we consider the integral

$$
\tilde{w}_{\alpha, \mathbf{q}}(z)=\int \exp \left[\mathbf{i q}_{j} \cdot \mathbf{t}\right] w_{\alpha}\left(\sqrt{z^{2}+t^{2}}\right) \mathrm{d} \mathbf{t}
$$

for the scalar weight functions, $\alpha=0,1,2,3$, and analogous integrals for the $x, y, z$ components of the vector weight function. For scalar weight functions $w_{\alpha}, \alpha=0,1,2,3$, and for $z$-components of the vector functions $w_{\alpha, z}, \alpha=1,2$, the last integral becomes

$$
\begin{aligned}
\tilde{w}_{\alpha, \mathbf{q}}(z) & =2 \pi \int_{z}^{\sigma / 2} w_{\alpha}(R) J_{0}\left(|\mathbf{q}| \sqrt{R^{2}+z^{2}}\right) R \mathrm{~d} R \\
\tilde{w}_{\alpha, z, \mathbf{q}}(z) & =2 \pi \int_{z}^{\sigma / 2} w_{\alpha, z}(R) J_{0}\left(|\mathbf{q}| \sqrt{R^{2}+z^{2}}\right) R \mathrm{~d} R
\end{aligned}
$$

whereas all in-plane vector components, $\tilde{w}_{\alpha, j, \mathbf{q}}, j=x, y$ are equal to zero. Therefore,

$$
\lambda_{s j, \mathbf{q}}(z)=\sum_{\alpha=0,1,2,3} \int_{-\sigma / 2}^{\sigma / 2}\left(\Phi^{\prime}\left(z+z^{\prime}\right)\right)_{\alpha, \mathbf{q}} \tilde{w}_{\alpha, z, \mathbf{q}}\left(\left|z^{\prime}\right|\right) \mathrm{d} z^{\prime}+\sum_{\alpha=1,2} \int_{-\sigma / 2}^{\sigma / 2}\left(\Phi^{\prime}\left(z+z^{\prime}\right)\right)_{\alpha, \mathbf{q}} \tilde{w}_{\alpha, \mathbf{q}}\left(\left|z^{\prime}\right|\right) \mathrm{d} z^{\prime}
$$

where $\left(\Phi^{\prime}(z)\right)_{\alpha, \mathbf{q}}$ is the $\mathbf{q}$-th Fourier expansion coefficient of the partial derivative $\partial \Phi_{\text {ex }} / \partial n_{\alpha}$. The Fourier expansion coefficients of the scalar weighted densities are

$$
n_{\alpha, \mathbf{q}_{j}}(z)=\int_{-\sigma / 2}^{\sigma / 2} \mathrm{~d} z \tilde{w}_{\alpha, \mathbf{q}_{j}}\left(\left|z^{\prime}\right|\right) \rho_{s, \mathbf{q}_{j}}\left(z+z^{\prime}\right) \mathrm{d} z^{\prime}
$$

and a quite analogous equation can be written for the $z$-components of the vector weighted densities, while the $x$ - and $y$-components of the vector local densities are equal to zero. Equations (17)-(19) complete the description.

Let us summarize the proposed procedure. At the beginning of the calculations we evaluate the Boltzmann function expansion coefficients, $g_{\mathbf{q}}(z)$, from equation (13) and the functions $\tilde{w}_{\alpha, \mathbf{q}}(z)$; they are calculated only once and stored. Next, we begin iterations from the assumed Fourier expansion coefficients $\tau_{s j, \mathbf{q}}(z)$. At very low bulk densities we can apply the ideal gas approximation, $\tau_{s j, \mathbf{q}}(z)=\delta_{\mathbf{q} \mathbf{0}} \rho_{s b} / M$, where $\rho_{s b}$ is the bulk total segment density. At the first step we evaluate the expansion coefficients $\rho_{s j, \mathbf{q}}(z)=\sum_{\mathbf{q}_{a}+\mathbf{q}_{b}=\mathbf{q}} \tau_{s j, \mathbf{q}_{a}}(z) g_{\mathbf{q}_{b}}(z)$ and then the weighted density coefficients from equation (19). The expansion (12) is used to calculate the weighed densities, and those, in turn, to evaluate the values of $\partial \Phi_{\mathrm{ex}} / \partial n_{\alpha}(\mathbf{r})$, and, consequently the coefficients $\left.\left(\Phi^{\prime}(z)\right)\right)_{\alpha, \mathbf{q}}$ by integrating equation (13). Then, the coefficients $\lambda_{s j, \mathbf{q}}(z)$ are calculated from equation (19). These coefficients yield the function $\lambda(\mathbf{r})$, which is next used to calculate the coefficients $\gamma_{\mathbf{q}}(z)$, again integrating $\exp [-\beta \lambda(\mathbf{r})]$ according to equation (13). Finally, the calculation of $\tau_{s j, \mathbf{q}}(z)$ from equation (14) is preceded by the evaluation of the coefficients $G_{\mathbf{q}}^{l}(z)$ from equation (15). The calculations are repeated until a required accuracy of the procedure is reached.

Previous numerical calculations for atomic adsorbates [27-29] have indicated that if the diameter $\sigma$ is equal or greater than the length of the lattice vectors $\mathbf{a}_{1}$ and $\mathbf{a}_{2}$, then the Fourier expansion of the cavity function $\tau_{s j}(\mathbf{r})$ is very fast convergent and in many cases only the zeroth-order term, $\tau_{s j}(\mathbf{0})$, suffices to obtain a quite accurate representation of the local density. In such cases one needs to evaluate only very few coefficients $G_{\mathbf{q}}^{l}(z), \lambda_{s j, \mathbf{q}}(z)$ and $\gamma_{\mathbf{q}}(z)$.

\section{Results and discussion}

Model calculations were carried out for chains adsorbed in pores with the walls built of (100) fcc crystal surfaces, assuming the length of the unit lattice vector equal to $\sigma,\left|\mathbf{a}_{1}\right|=\left|\mathbf{a}_{2}\right|=\sigma$. We have also assumed that $\sigma_{g s}=\sigma$. Similarly to the case of the potential expansion (3), all Fourier expansion coefficients $\chi_{\mathbf{q}}(z)$ depend only on the length of the vector, $q=|\mathbf{q}|$. The Fourier expansions can be simplified by introducing the functions $f_{l}(x, y)=\sum^{\prime} \exp [-\mathbf{i q} \cdot \mathbf{t}]$, where the 
summation runs over all reciprocal vectors of a given length, $q_{l}=\left|\mathbf{q}_{l}\right|$. Of course, the functions $f_{l}(x, y)$ are identical to those used in the expansions of the external potential (3) and they can be found in [30].

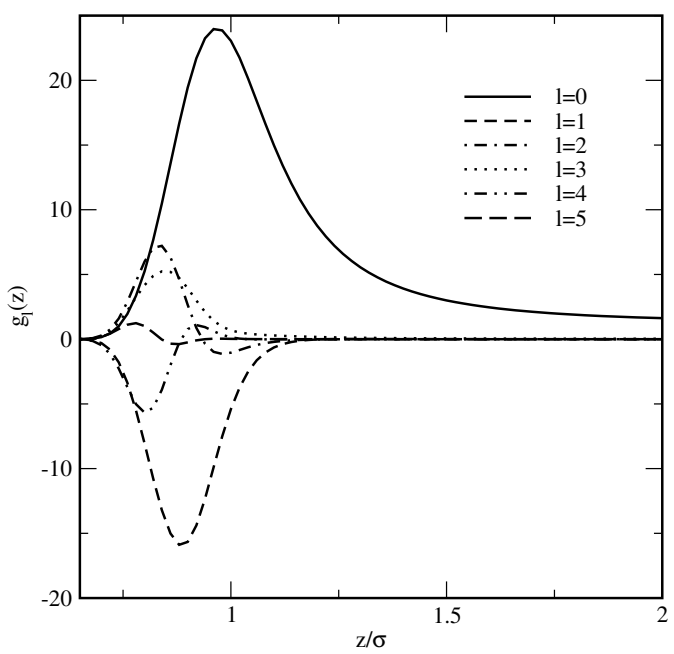

Figure 1. Fourier expansion coefficients for the Boltzmann function. The values of all parameters are given in the text.

In figure 1 we present the first six Fourier Boltzmann function coefficients $g_{l}(z)$ obtained for $k T / \varepsilon_{g s}=0.25$ and for $H=20 \sigma$. We see that the magnitude of $g_{5}(z)$ is quite small compared to the coefficient $g_{0}(z)$. Therefore, the Fourier expansion of the Boltzmann function can be truncated after $l=5$ term.

In the case of chains built of up to 16 segments and for the pore considered above, we have also tested the convergence of the Fourier expansion for one-particle background (or cavity) function $\tau(\mathbf{r})$. We found that even for bulk total segment densities as high as $\rho_{s b} \sigma^{3}=0.6$ the truncation of this expansion after the term $\tau_{1}(z)$ was sufficient to obtain quite accurate results. Therefore, all further calculations were carried out assuming that the Fourier expansions of the Boltzmann function and of the cavity function can be truncated after $l=5$ and $l=1$ terms, respectively.
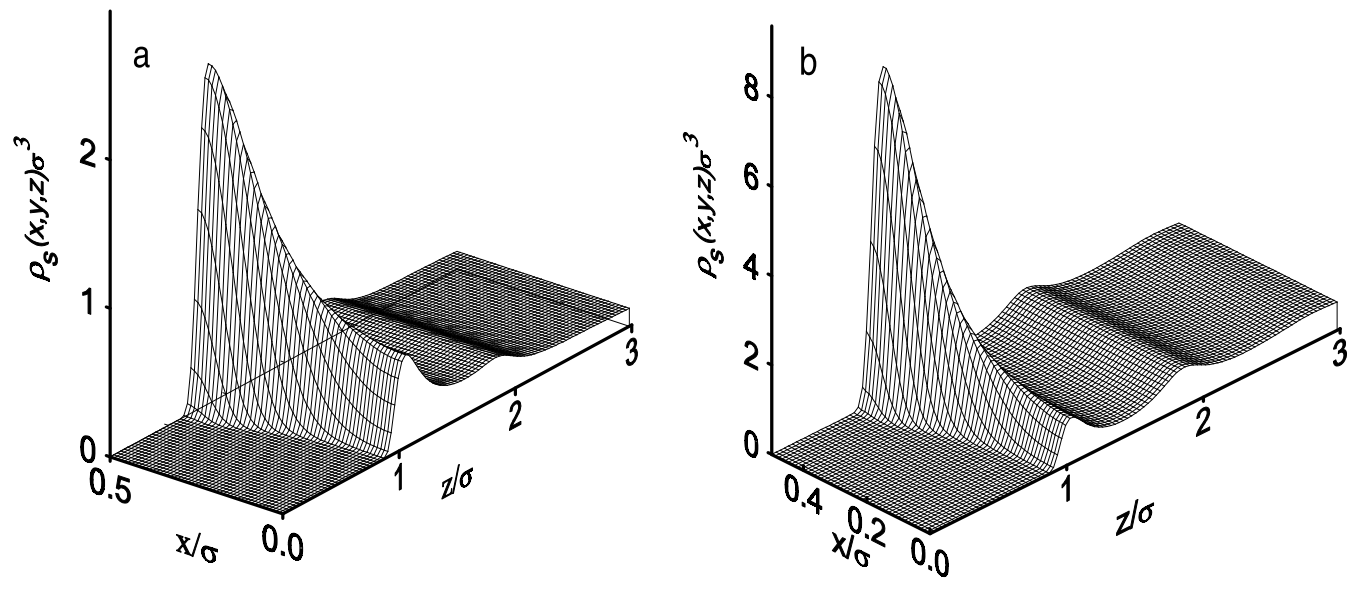

Figure 2. The change of the total segment densities of 8 -mers along the diagonal of the unit lattice cell, $\rho_{s}(x, x, z)$ at the pore wall located at $z=0$. Part a is for $\rho_{s b} \sigma^{3}=0.1$, whereas part $\mathrm{b}-$ for $\rho_{s b} \sigma^{3}=0.6$. The pore width is $H=20 \sigma$ and $k T / \varepsilon_{g s}=0.3$. 
We begin with the presentation of the results illustrating the effect of the periodicity of the adsorbing potential on the structure of adsorbed fluid. In figure 2 we show the changes of the total segment local density of 8-mers with the distance from the pore wall, $z$, along the diagonal of the unit lattice crystal cell, $x=y$. Again, the calculations were carried out for $H=20 \sigma$. Part a is for the total segment bulk density $\rho_{s b} \sigma^{3}=0.1$ and part $\mathrm{b}-$ for $\rho_{s b} \sigma^{3}=0.6$. One observes that for both bulk densities the periodicity of the adsorbing potential mainly affects the behavior of the first fluid layer adjacent to the pore wall. Indeed, the in-plane changes of the density profile for the second adsorbed layer are hardly seen on the scale of figure 2. Instantaneously, an increase of the bulk density $\rho_{s b}$ enhances the accumulation of segments located within the first adsorbed layer over the so-called adsorbing site position, $\mathrm{S},(x / \sigma=1 / 2, y / \sigma=1 / 2)$.
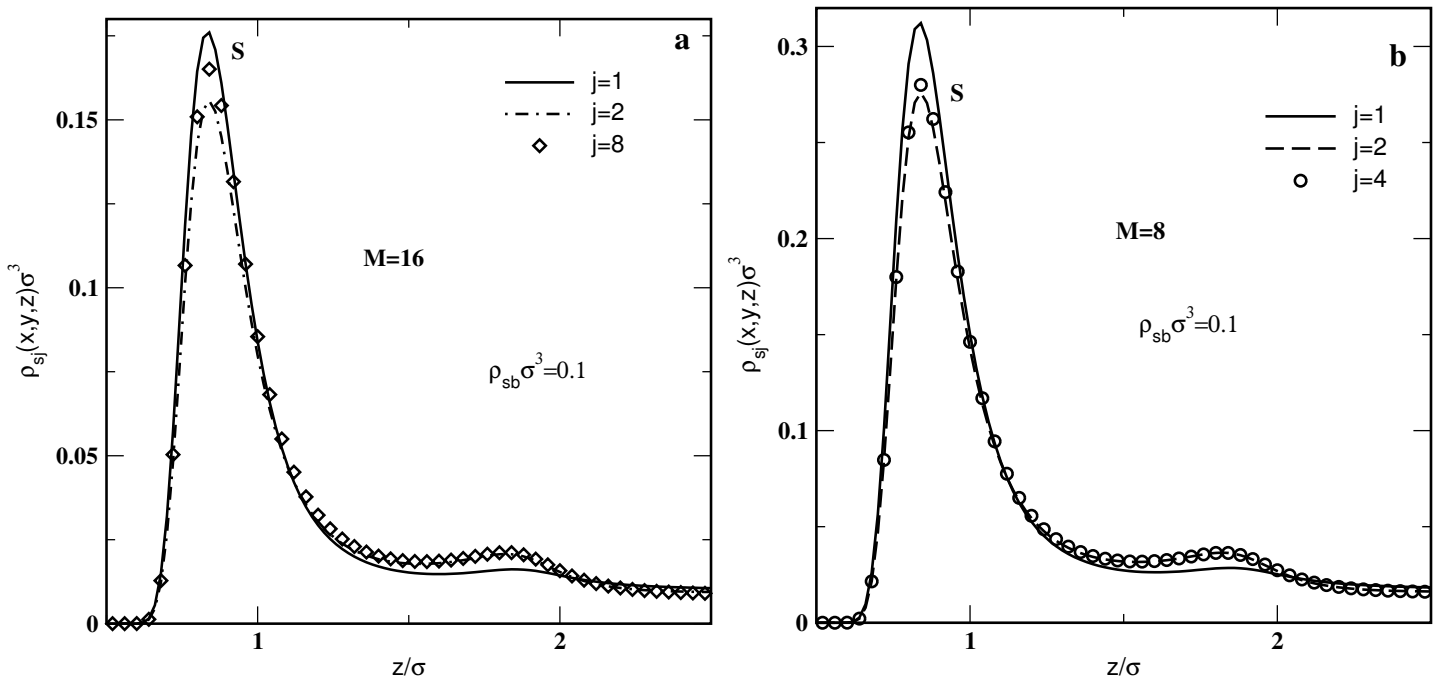

Figure 3. The density profiles of segments $j$ over the $\mathrm{S}$ position of the unit lattice cell of the pore wall at $z=0$. Part a is for adsorbed 16-mers and part $\mathrm{b}$ - for adsorbed 8-mers. The bulk density is $\rho_{s b} \sigma^{3}=0.1$. The pore width is $H=20 \sigma$ and $k T / \varepsilon_{\text {gs }}=0.3$.

More information on the structure of an adsorbed layer is provided by the inspection of densities of consecutive segments. In figure $3 \mathrm{a}$ we show the changes of the profiles of the segments $j=1,2$ and 8 of 16 -mers over the position $\mathrm{S}$. The results presented in figure $3 \mathrm{~b}$ were obtained for 8 -mers adsorbed over the same position of the crystal surface and for $j=1,2$ and 4 . The bulk total segment density is equal to $\rho_{s b} \sigma^{3}=0.1$ in both cases. It means that the bulk density of individual segments in the system of 16-mers is two times lower than in the system involving 8-mers. Consequently, the local densities of individual segments of 16-mers are lower than the local densities in the system of 8-mers. For both chain lengths the local density of the first segment, $j=1$, exhibits the highest maximum. The first segment is preferentially adsorbed over the $\mathrm{S}$ position. Thus, the local density of the adjacent segment, $j=2$, over the same position should be less probable. In fact, the maximum of the function $\rho_{s 2}(\sigma / 2, \sigma / 2, z)$ is the lowest in comparison with the maxima of all other segments. For the adsorbed 8-mers the differences between the maxima for $j=2$ and for $j=4$ (the middle segment) are small, whereas for the adsorbed 16-mers these differences are slightly more pronounced. We also note that the second layer density profiles for all, but $j=1$, segments are almost identical. The second maximum of $\rho_{s 1}(\sigma / 2, \sigma / 2, z)$ is lower than all the remaining second maxima.

A comparison of the total segment profiles of 16- and 8-mers over three positions of the crystal lattice, i.e., over the $\mathrm{S}$ position, over the $\mathrm{SP}$ position (i.e. the saddle point position, $x=1 / 2, y=0$ ) and over an atom of the crystal surface, $\mathrm{A},(x=0, y=0)$ evaluated at three bulk densities, $\rho_{s b}=0.1,0.2$ and 0.4 is shown in figure 4 . Comparing the results shown in figure 3 with those given in figure 4 one should have in mind that the total density profile is the sum of the local densities of individual segments. The first maxima of the profiles of 16-mers are higher than the 


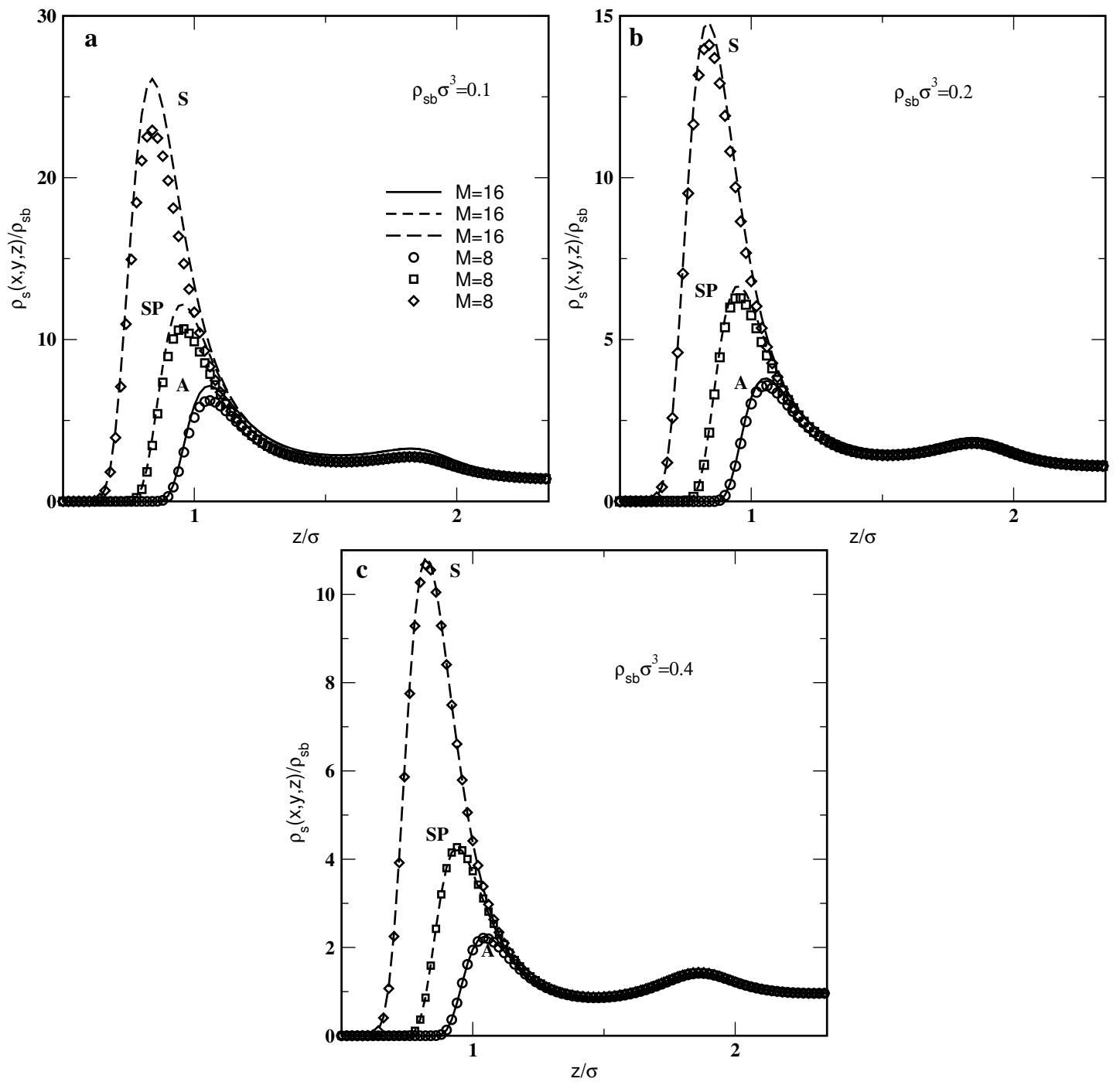

Figure 4. A comparison of the total segment density profiles, $\rho_{s}(x, y, z)$ of 16-mers (lines) and 8 -mers (symbols) over the positions S, SP and A of the pore wall at $z=0$ and at three bulk densities, $\rho_{s b} \sigma^{3}=0.1$ (part a), 0.2 (part b) and 0.4 (part c). The pore width is $H=20 \sigma$ and $k T / \varepsilon_{g s}=0.3$.

maxima of the profiles of 8-mers. This is particularly well seen at $\rho_{s b}=0.1$ and for the profiles over the S-position. At low bulk densities, the attractive fluid-solid interactions determine the adlayer structure. If there is enough free space on the surface, all the chains can assume an in-plane orientation and the effect of the promotion of some segments to higher layers is small. Moreover, because of bonding, location of the $j-1$ and $j+1$ segments on the wall enforces a similar location of the segment $j$ and this effect is more important for longer chains. With the bulk density increase the differences between the total segment profiles of 16-mers and of 8-mers become smaller. This is due to the fact that with the density increase, the hard-sphere and the segment-surface interaction contributions to the free energy functional prevail and the contribution arising from the chain connectivity plays a smaller role in determining the structure of the fluid adjacent to the wall. Obviously, with the bulk density increase we observe the formation of a multilayer structure at the wall and at $\rho_{s b}=0.2$, the second local density maximum is developing. However, the shape and the location of the second maximum is almost independent of the position over the crystal lattice. 
Figure 5 compares the segment densities of the selected individual segments, i.e. the first $(j=1)$ and of the middle ( $j=4$ for 8 -mers and $j=8$ for 16 -mers) segments over the S, SP and A positions of the crystal lattice. The first segments are preferentially adsorbed at the S-position and an increase of the bulk density amplifies this tendency. Although it is not quite well seen on the figure scale, we can state that the middle segment, compared to the first segment "prefers" the A-position. Over the $\mathrm{S}$ and $\mathrm{SP}$ positions this tendency is opposite.
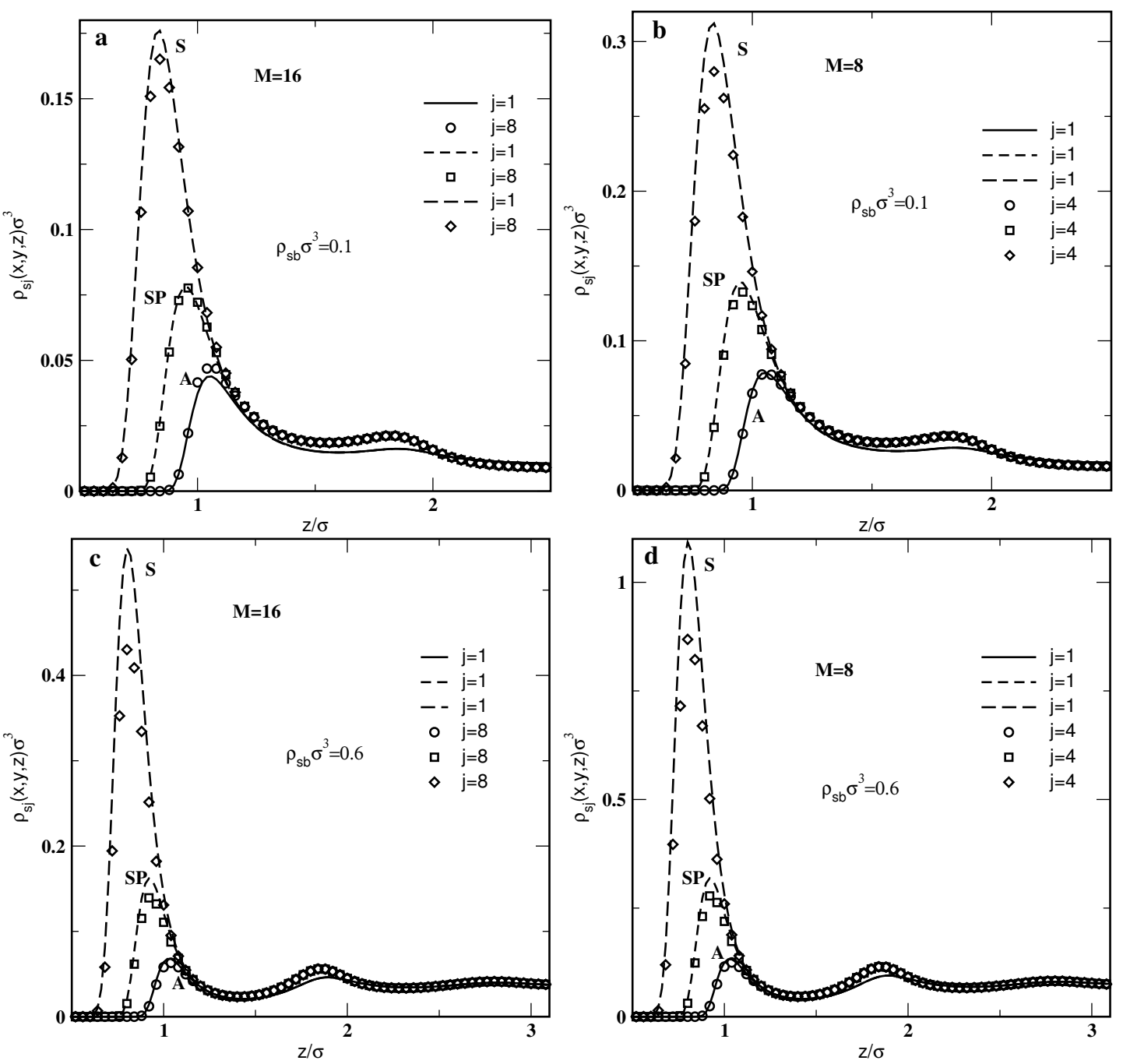

Figure 5. A comparison of the density profiles of the first (lines) and of the middle (symbols) segments of 16-mers (pars a and c) and of 8-mers over the positions S, SP and A of the pore wall at $z=0$. Parts a and $\mathrm{b}$ are for $\rho_{s b} \sigma^{3}=0.1$, whereas parts $\mathrm{c}$ and $\mathrm{d}-$ for $\rho_{s b} \sigma^{3}=0.6$. The pore width is $H=20 \sigma$ and $k T / \varepsilon_{g s}=0.3$.

The numerical calculations performed in this work clearly demonstrate the effect of a periodic variations of the adsorbing potential on the structure of the adsorbed layer. We now consider how the energy periodicity of the pore walls effects the adsorption of a fluid in pores and the solvation force. The amount of the confined fluid per unit surface area is given by

$$
\Gamma=\frac{1}{\mathbf{a}_{1} \times \mathbf{a}_{2}} \iint_{\mathbf{a}_{1} \times \mathbf{a}_{2}} \rho_{s}(\mathbf{r}) \mathrm{d} \mathbf{r}=\int_{0}^{H} \rho_{s \mathbf{0}}(z) \mathrm{d} z .
$$

We recall that $\rho_{s \mathbf{0}}(z)$ is the zeroth-order expansion coefficient of the total segment density profile. The calculation of the solvation force is carried out differentiating the grand potential $\Omega=F-$ 
$\int \mathrm{d} \mathbf{r}[\mu-v(\mathbf{r})] \rho_{s}(\mathbf{r})$

$$
F_{s}=-\frac{\partial \Omega}{\partial H}-p
$$

where $p$ is the pressure of a bulk fluid at the total segment density $\rho_{s b}$. It can be easily proven [21] that the grand thermodynamic potential $\Omega$ is conveniently evaluated from

$$
\Omega=\sum_{i=1}^{M} \int \mathrm{d} \mathbf{r} \lambda(\mathbf{r}) \rho_{s i}(\mathbf{r})+\int \mathrm{d} \mathbf{r} \Phi_{\mathrm{ex}}(\mathbf{r})+k T \Gamma .
$$

The solvation force is an experimentally accessible quantity in measurements using surface force apparatus [32]. Several groups investigated the dependence of solvation force on the specific properties of confining solid substrates, namely the shape of the surface, its symmetry, some aspects of energy and geometrical heterogeneity, cf. [33-35].
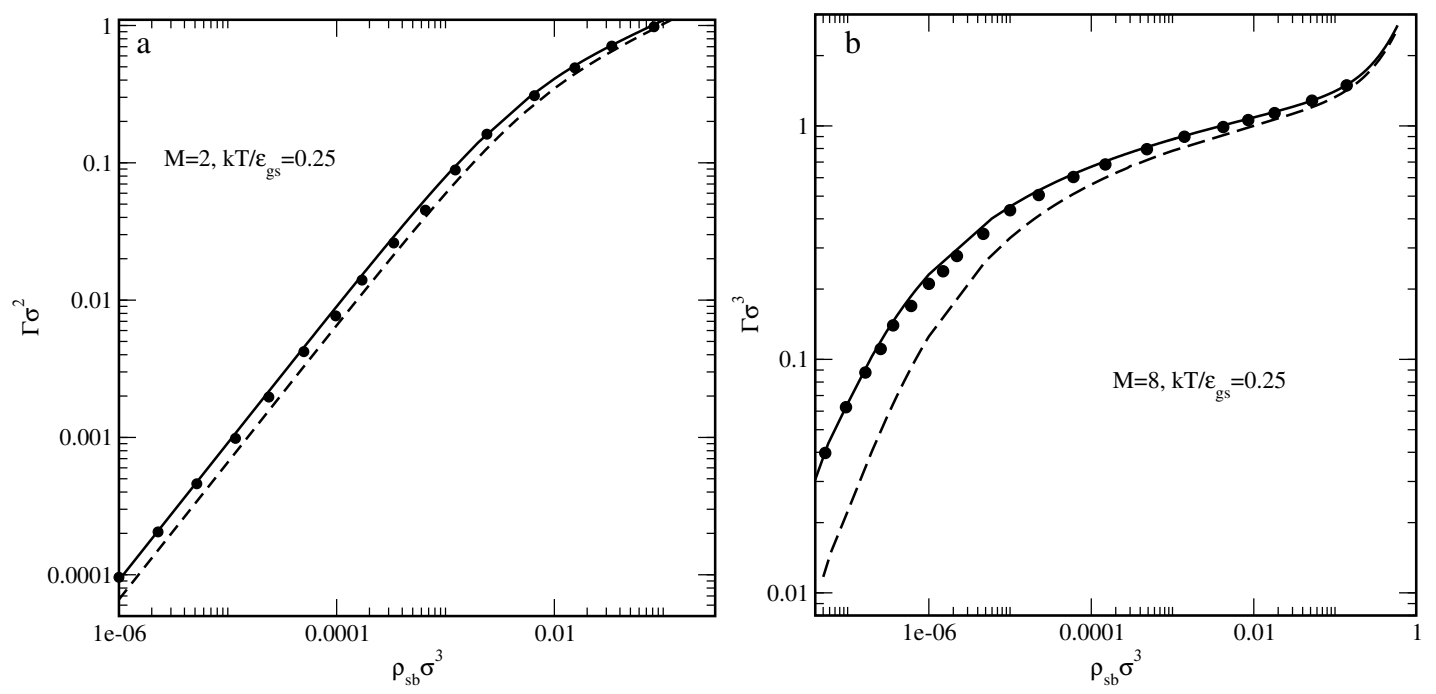

Figure 6. A comparison of the adsorption isotherms of dimers (part a) and 8-mers (part b). Solid lines are the isotherms for pores with crystalline walls, dashed lines - for pores with the walls interacting via the potential $v_{\mathbf{0}}(z)$, whereas symbols have been obtained for the Boltzmannaveraged potential from equation (23). The pore width is $H=20 \sigma$.

In figure 6 we show the adsorption isotherms obtained for dimers (part a) and for 8-mers (part b). Solid lines denote the isotherms calculated for crystalline pore walls, assuming that $k T / \varepsilon_{g s}=0.25$. Dashed lines were evaluated for energy homogeneous surfaces. In this case the adsorbing potential was equal to the zeroth order term in the Fourier expansion $(4), v(z)=$ $v_{\mathbf{0}}^{\prime}(z)+v_{\mathbf{0}}^{\prime}(H-z)$. The isotherms for the latter model are lower than those evaluated assuming periodic modulation of the adsorbing potential. The differences between the isotherms for uniform and for crystalline pore walls increase with an increase of the chain length. The latter effect is understandable, because the total fluid-pore potential energy increases with an increase of the number of segments of a chain molecule. However, if we define the reference adsorbing potential by

$$
v_{\text {ref }}(z)=-k T\langle\exp [-v(\mathbf{r}) / k T]\rangle_{\mathbf{q}=\mathbf{0}},
$$

then the isotherms obtained assuming that the adsorbing potential is given by $v_{\text {ref }}(z)$ become close to those evaluated for crystalline pore walls. The "Boltzmann-averaged" potential (23) guarantees that in the limit of low bulk densities, the isotherms for crystalline and uniform walls are identical. Instantaneously, our calculations indicate that approximation of the adsorbing potential by equation (23) is reasonably accurate for dimers and longer chains, cf. figures (6a) and (6b). In fact, the effects of energy heterogeneity of an adsorbing surface are less important for bigger adsorbate 
molecules, i.e. longer chains in our case. We also note that the adsorption is higher for longer molecules, especially at low bulk densities. For very low bulk densities this is quite obvious. Within this region of densities, the attraction between molecules and the wall plays a predominant role. Thus, higher attraction for longer chains results in higher adsorption. This behavior was already observed in our previous works [36-38].

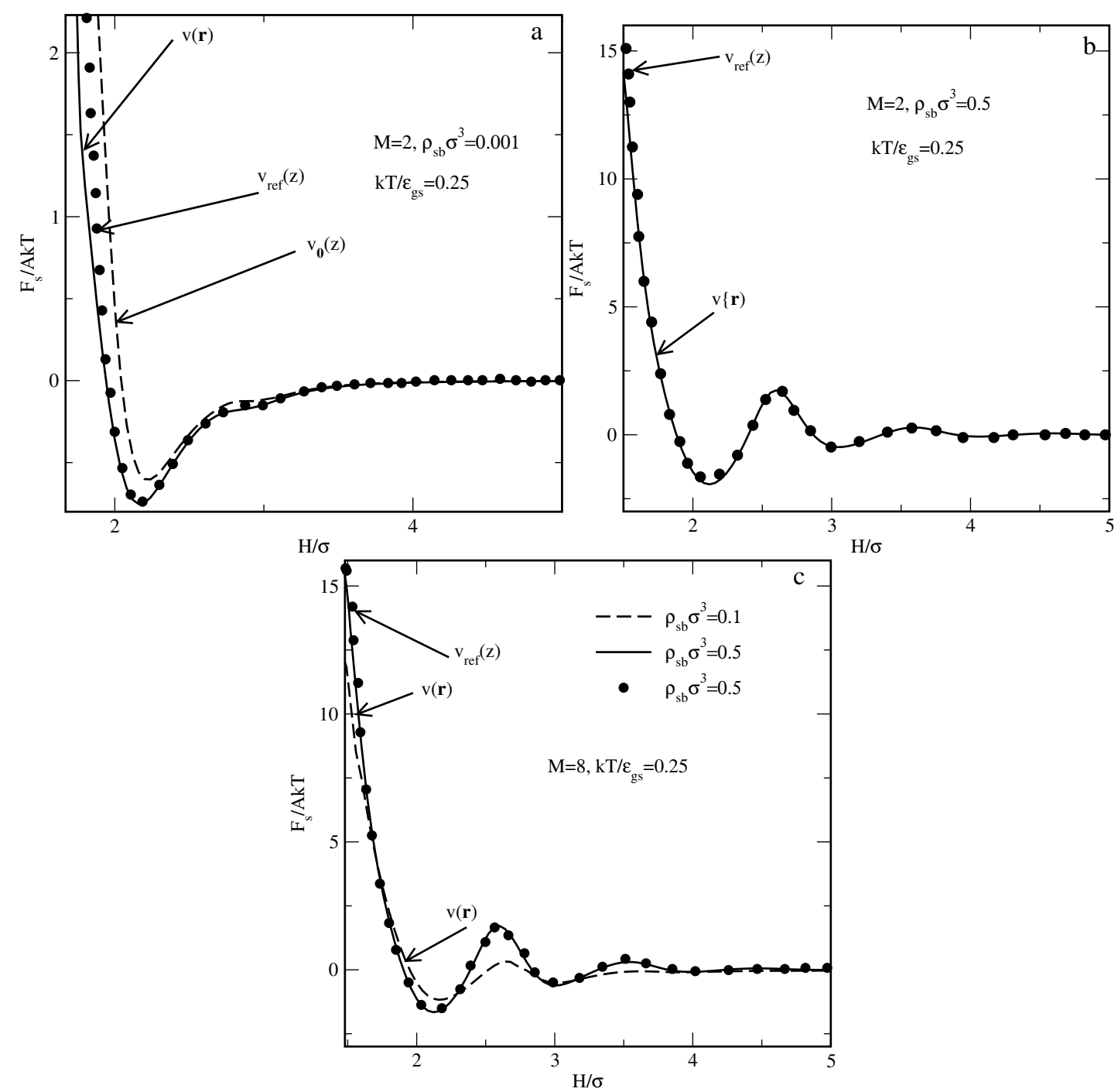

Figure 7. The plots of the solvation force per unit surface area $A$. Parts a and $\mathrm{b}$ are for dimers, part $\mathrm{c}$ - for octamers. All the parameters are given in figures. The labels $v_{\mathbf{0}}(z), v_{\mathrm{ref}}(z)$ and $v(\mathbf{r})$ refer to external field potential models.

Figures $7 \mathrm{a}-7 \mathrm{c}$ present the plots of the solvation force. The calculations were carried out for dimers (parts a and b) and octamers (part b) confined between crystalline walls (the curves abbreviated by the symbol $v((r)))$ and between uniform walls interacting via the potential $v_{\text {ref }}(z)$, equation (23). In the case of dimers at low bulk density, we have also included the plot of the solvation force obtained assuming that the segment-pore walls interaction potential is given by the first term in the Fourier expansion (4), cf. figure 7a. Part a was evaluated at low bulk density, i.e. low amount of confined fluid, whereas parts b and c were calculated for intermediate and high bulk fluid densities.

At small wall-to-wall distances, the solvation force is strongly repulsive. Obviously, with an increase of the bulk density, the plots of the solvation force exhibit more oscillations. When the bulk 
density increases (i.e. the amount of confined chains increases) the differences between solvation force calculated for the crystalline walls and for the reference potential $v_{\text {ref }}(z)$ decrease. Thus, in several cases the results for a crystalline solid can be well approximated by those evaluated for an uniform surface, exhorting the potential $v_{\text {ref }}(z)$. The possibility of using the reference potential $v_{\text {ref }}(z)$ significantly simplifies the calculations. One should remember, however, that the potential $v_{\text {ref }}(z)$ is temperature-dependent.

Our work has concentrated on methodological aspects and its aim was to develop a suitable numerical method for studying adsorption of chain particles on crystalline surfaces. Of course, the approach presented here can be further used to investigate the phase transitions in the films containing chain particles adsorbed on crystalline surfaces or in pores with crystalline walls.

We would also like to emphasize that the model of fluid-solid interaction considered in this work can be treated as an intermediate one between the model of a flat (energy homogeneous) wall and the model introduced by Tripathi and Chapman [39], that described adsorption of associating fluids on the surfaces activated with polar sites to which fluid molecules can bond or associate. This model was then generalized to the case of adsorption of chain molecules by Tscheliessnig et al. [40], that considered the case when associative surface sites were capable of bonding the "heads" of chain particles. In the limit of a complete association, the latter model seems to be suitable for the description of the formation of "brushes".

\section{Acknowledgements}

This project has been partially supported by the National University of Mexico under grant IN-223808-2. O.P. is on sabbatical leave from Instituto de Quimica de la UNAM.

\section{References}

1. Jiang J.W., Sandler S.I., Fluid Phase Equilibr., 2005, 228, 189.

2. Piccolo L., Loffreda D., Aires F.J.C.S., Deranlot C., Jugnet Y., Sautet P., Bertolini J.C., Surface Sci., 2004, 566, 995.

3. Engkvist O., Stone A.J., J. Chem. Phys., 2000, 112, 6827.

4. Curtarolo S., Stan G., Bojan, M.J., Cole M.W., Steele W.A., Phys. Rev. E, 2000, 61, 1670.

5. Minot C., Van Hove M.A., Biberian J.-P., Surf. Sci., 1996, 346, 283.

6. Marx D., Wiechert H., Adv. Chem. Phys., 1996, 95, 213.

7. Hansen F.Y., Bruch L.W., Taub H., Phys. Rev. B, 1995, 52, 8515.

8. Hansen F.Y. Bruch L.W., Phys. Rev. B, 1995, 51, 2515.

9. Marx D., Opitz O., Nielaba P., Binder K., Phys. Rev. Lett., 1993, 70, 2908.

10. Vakarin E., Duda Y., Holovko M., J. Chem. Phys., 1997, 107, 5569.

11. Holovko M., Vakarin E., Mol. Phys., 1996, 87, 1375.

12. Zeppenfeld, P., George J., Diercks V., Halmer R., David R., Comsa G., Marmier A., Ramseyer C., Girardet C., Phys. Rev. Lett., 1997, 78, 1504.

13. Hoang P.N.M., Picaud S., Girardet C., Meredith W., J. Chem. Phys., 1996, 105, 8453.

14. Vigiani A., Cardini G., Schettino V., J. Chem. Phys., 1997, 106, 5693.

15. Bruch L.W., Hansen F.Y., Phys. Rev. B, 1998, 57, 9285.

16. Trabelsi M., Coulomb J.P., Degenhardt D., Lauter H., Surf. Sci., 1997, 377-379, 38.

17. Mouritsen O.G., Berlinsky A.J., Phys. Rev. Lett., 1982, 48, 181.

18. Sokołowska Z., Sokołowski S., J. Colloid Interface Sci., 1988, 123, 73.

19. Łajtar L., Patrykiejew A. Sokołowski S., Surface Sci., 1988, 200, 288.

20. Wertheim M.S., J. Chem. Phys., 1987, 87, 7323.

21. Yu Y.-X., Wu J., J. Chem. Phys., 2002, 117, 2368.

22. Yu Y.-X., Wu J., J. Chem. Phys., 2003, 118, 3835.

23. Bryk P., Pizio O., Sokołowski S., J. Chem. Phys., 2005, 122, 194904.

24. Wu J., Li Z., Ann. Rev. Phys. Chem., 2007, 58, 85

25. Wu J., AIChE J., 2006, 52, 1169.

26. Li Z., Cao D., Wu J., J. Chem. Phys., 2005, 122, 224701.

27. Sokołowski S., Steele W.A., Langmuir, 1985, 1, 190.

28. Henderson D., Sokołowski S., Pizio O., J. Chem. Phys., 1995, 102, 9048. 
29. Pizio O., Sokołowski S., Phys. Rev. E, 1996, 53, 820.

30. Steele W.A., Surface Sci., 1973, 36, 317.

31. Rosenfeld Y., Phys. Rev. Lett., 1989, 63, 980.

32. Israelachvili J.N., Adams G.E., J. Chem. Soc. Faraday Trans. I, 1978, 74, 975.

33. Ghatak C., Ayappa K.G., J. Chem. Phys., 2004, 120, 9703.

34. Henderson J.R., Molec. Simul., 2005, 31, 435.

35. Sprenger M., Schlesener F., Dietrich S., J. Chem. Phys., 2006, 124, 134703.

36. Bryk P., Sokołowski S., J. Chem. Phys., 2004, 121, 11314.

37. Bryk P., Pizio O., Sokołowski S., J. Chem. Phys., 2005, 122, 194904.

38. Bryk P., Bucior K., Sokołowski S., Żukociński G., J. Phys. Chem. B, 2005, 109, 2977.

39. Tripathi S., Chapman W.G., J. Chem. Phys., 2003, 119, 12611.

40. Tscheliessnig R., Billes W., Fischer J., Sokołowski S., Pizio O., J. Chem. Phys., 2006, 124, 164703.

\title{
Адсорбція ланцюгових молекул в порах з кристалічними стінками: підхід функціоналу густини
}

\author{
К.Буцьор ${ }^{1}$, А.Патрикейев ${ }^{1}$, С.Соколовскі ${ }^{1}$, О.Пізіо ${ }^{2}$ \\ 1 Факультет моделювання фізико-хімічних процесів, Університет Марії Кюрі-Склодовської, Люблін, \\ Польща \\ 2 Автономний університет метрополії, Ізтапалапа, Мехіко, Мексика
}

Отримано 29 листопада 2008 р., в остаточному вигляді - 7 лютого 2009 р.

Розвинуто мікроскопічну теорію функціоналу густини для вивчення адсорбції ланцюгових молекул на притягальних твердих поверхнях кристалічної симетрії. Запропонований підхід ґрунтується на розкладах одночастинкових функцій у двовимірні ряди Фур'є. Для ілюстрації проведено обчислення для димерів, 8- і 16-мерів, що адсорбовані на кристалічній поверхні (100) fсс, які демонструють вплив періодичності адсорбуючого потенціалу на структуру адсорбованого шару і термодинаміку адсорбції.

Ключові слова: функціонал густини, адсорбція, ланцюги, кристали

PACS: 68.08.-p, 68.43.Fg, 82.35.Gh, 68.43.-h 